$\mathrm{D}$ Von Hartwig Berger e Klimaschutzpolitik steht vor einer schwierigen Phase. Innerhalb der Europäischen Union drohen elf der 15 jetzigen Mitgliedstaaten ihr Treibhausgasreduktionsziel bis 2010 deutlich zu verfehlen. Auch in Deutschland sind die Jahre sinkender Kohlendioxid-Emissionen vorbei. Im vergangenen Jahr nahmen sie trotz schwachem Wirtschaftswachstum sogar um einen halben Prozentpunkt zu. Die Erfüllung der Zielverpflichtung von minus 25 Prozent bis 2005, gerechnet auf das Basisjahr 1990, ist ernsthaft in Frage gestellt. Eine kritische Überprüfung der deutschen Klimaschutzpolitik und ihrer Maßnahmen ist das Gebot der Stunde und im Rahmen Klimaschutzprogramms von 2000 für dieses Jahr auch gefordert.

Die gegenwärtige Debatte um die Steuerreform böte dazu einen hervorragenden Einstieg. Es mehren sich Stimmen, die den Abbau umweltschädlicher Subventionen vorschlagen. Der Abschied von der Zersiedlungsprämie Eigenheimzulage ist langfristig wirkender Klimaschutz, zumal dann, wenn ein Teil der so eingesparten Gelder als Förderprogramm zur Altbausanierung eingesetzt wird. Die vom Umweltbundesamt angeregte entsprechende Umwidmung der Steinkohlesubventionen des Bundes - 3,1 Milliarden Euro jährlich -könnte nach unseren Berechnungen 90.000 Arbeitsplätze schaffen und 17 Millionen Tonnen Kohlendioxid dauerhaft reduzieren.

\section{Sorgenkind Verkehr}

Die Entwicklung des motorisierten Verkehrs ist unstrittig eine Achillesferse des Klimaschutzes. 11,2 Prozent Kohlendioxid-Zuwachs von 1990 bis 1998 plus prognostizierte Steigerungsraten fuir die Zukunft - das Alarmzeichen ist unübersehbar. Für eine Überarbeitung der Bundespolitik ist der Luftverkehr ein wichtiger Hebel, immerhin verantwortlich für 25 bis 30 Prozent der verkehrsbedingten Klimawirkung. Die Aufhebung der Mehrwertsteuerbefreiung wäre ein erster notwendiger Schritt. Deutschland sollte auch eine EU-Initiative zur Aufhebung der Flugbenzinsteuer-Befreiung starten. Die neue EU-Richtlinie zur Energiebesteuerung lässt ein koordiniertes Vorangehen mehrerer Mitgliedsländer ausdrücklich zu. Ebenso sollte die Bundesregierung die Empfehlung des Wissenschaftlichen Beirats für globale Umweltfragen (WBGU) vom Frühjahr 2003 aufgreifen. Der WBGU schlägt einen inter-

Die Überarbeitung des deutschen Klimaschutzprogramms steht an

\title{
Wolken überm Klimahimmel
}

\section{Dieses Jahr, so sieht es das Kli-} maschutzprogramm von 2000 vor, muss die Bundesregierung ihre Klimaschutzpolitik überdenken. Angesichts steigender Emissionen und Fehlleistungen in vielen Bereichen, muss sie endlich zu drastischen Maßnahmen greifen. Eine öffentliche Diskussion darüber ist bis dato aber nicht aufgekommen.

nationalen Vorstoß für ein emissionsabhängiges Nutzungsentgelt im Luftverkehr vor, dessen Erträge für Klimaschutz und Umweltmaßnahmen in Entwicklungsländern eingesetzt wird. Die Einbeziehung weiterer Länder in die internationalen Klimaschutzverhandlungen würde dadurch erleichtert. Weitere Ansatzpunkte beim Verkehr sind eine Revision des Verkehrwegeplans und eine gesetzliche Auflage zur Reduzierung des Treibstoffverbrauchs von Fahrzeugen.

Im Strom- und Wärmesektor ist das Scheitern der freiwilligen Vereinbarung zur Förderung der Kraft-Wärme-Kopplung bereits jetzt evident. Die Bonus-Regelungen für KWK-Anlagen werden durch niedrigere Einspeisevergütungen unterlaufen. Die Bundesregierung sollte sich daher zur Einfuihrung der seinerzeit von Wirtschaftsminister Müller blockierten Quotenregelung entschließen oder eine gesetzliche Einspeisevergütung analog dem Erneuerbare-Energien-Gesetz erlassen. Nur so ist das erklärte Ziel, bis 201023 Millionen Tonnen an Kohlendioxid bei der Kraft-WärmeKopplung einzusparen, noch erreichbar. Ausbaufähig ist der Sektor Energie-Effizienz insgesamt. Strategisch wichtig ist hier ein Engagement auf europäischer Ebene zum Erlass einer Energie-Effizienz-Richtlinie. Eine europäische Konferenz von Experten und Akteuren hat dazu im März 2003 mehrere Vorschläge ausgearbeitet. Im Kern laufen sie darauf hinaus, Energieunternehmen zur Einrichtung eines Fonds zu verpflichten, aus dem solche Maßnahmen finanziert werden können.
Die strategische Bedeutung eines Ausbaus der Erneuerbaren Energien für Klimaschutz ist unstrittig. Dringend ist hier die Fortschreibung der gesetzlichen Einspeisevergütung, die derzeit das Wirtschaftsministerium unter dem Einfluss der großen Stromunternehmen verzögert und behindert. Wichtig sind zudem Impulse für den Ausbau der Solarwärme, die im bisherigen Klimaschutzprogramm keine Berücksichtigung finden. Eine so einfache wie weitreichende Lösung ist die Einführung einer Ausgleichsabgabe, die alle Erzeuger von fossiler Wärme entrichten müssen und deren Erträge zum Ausbau der Solarwärme eingesetzt werden.

\section{Stiefkind Landwirtschaft}

Als wichtiges Handlungsfeld muss das Klimaschutzprogramm die Landwirtschaft mehr als bisher nutzen. Das alte Konzept atmet noch den Geist eines konventionell denkenden Ministeriums. Hier sollte die neue Agrarpolitik Wirkung zeitigen - schließlich reduziert Bio-Anbau auch Klimawirkungen durch einen geringeren Energieeinsatz, maßvolle Viehhaltung und den Verzicht auf nitrathaltige Dünger.

Zu Unrecht wird ein Handlungsfeld in der Klimaschutzdebatte zumeist übersehen: Die fluorierten Gase, die als Substitut für den Ozonkiller FCKW zunehmend als Isoliermaterial, Schäumund Kühlmittel zum Einsatz kommen. Sie machen zwar nur wenige Prozent des nationalen Klimagas-Budgets aus, würden aber in Fortsetzung jetziger Trends um ein Mehrfaches anwachsen. Das alte Klimaschutzprogramm hatte hier auf wirksame Maßnahmen verzichtet. Hier kann und sollte nachgebessert werden, zumal Substitute für Fluorgase technisch verfügbar und auch wirtschaftlich einsetzbar sind.

Wenn Klimaschutz ernsthaft verfolgt wird, kann die Kyoto-Verpflichtung nur ein Anfang sein. Zusätzlich zu einem befristeten Programm brauchen Deutschland und Europa daher eine Debatte um Klimaschutzziele und weiterreichende Maßnahmen über das Jahr 2010 hinaus. Deutschland sollte sich hier an den Empfehlungen der einschlägigen Enquète-Kommissi-

\section{Der Autor}

Dr. Hartwig Berger ist Privatdozent am Institut für Soziologie der FU Berlin.

Kontakt: Weimarer Str. 31, 10625 Berlin, Tel. 03031800406, E-Mail: hartwig.berger@t-online.de 
(c) 20I0 Authors; licensee IÖW and oekom verlag. This is an article distributed under the terms of the Creative Commons Attribution Non-Commercial No Derivates License (http://creativecommons.org/licenses/by-nc-nd/3.o/), which permits unrestricted use, distribution, and reproduction in any medium, provided the original work is properly cited. 